\title{
UNILATERAL PYODERMA GANGRENOSUM OF THE EXTERNAL EAR IN A RESOURCE LIMITED SETTING: CASE REPORT AND LITERATURE REVIEW
}

Zephania Abraham ${ }^{1}$, Edwin Thomas ${ }^{1}$, and Aveline Kahinga ${ }^{2}$

${ }^{1}$ University of Dodoma College of Health and Allied Sciences

${ }^{2}$ Muhimbili University of Health and Allied Sciences School of Medicine

November 30, 2020

\author{
Abstract \\ Pyoderma gangrenosum (PG) being a primary sterile neutrophilic dermatosis, the etiology is still uncertain and it rarely affects \\ children. A high index of suspicion is essential to execute its prompt treatment. To the best of our knowledge this is the first \\ reported case in our country \\ UNILATERAL PYODERMA GANGRENOSUM OF THE EXTERNAL EAR IN A RE- \\ SOURCE LIMITED SETTING: CASE REPORT AND LITERATURE REVIEW \\ Zephania Saitabau Abraham ${ }^{1}$ Edwin Peter Thomas ${ }^{1}$ Aveline Aloyce Kahinga ${ }^{2}$ \\ ${ }^{1}$ Department of Surgery-University of Dodoma, College of Health and Allied Sciences, Dodoma-Tanzania \\ Box 259, Dodoma \\ ${ }^{2}$ Department of Otorhinolaryngology-Muhimbili University of Health and Allied Sciences, Dar es Salaam, \\ Tanzania \\ Box 65001, Dar es Salaam-Tanzania \\ Correspondence \\ Dr. Zephania Saitabau Abraham \\ Department of Surgery- University of Dodoma, College of Health and Allied Sciences \\ Email: zsaaitabau@yahoo.com
}

\section{ABSTRACT}

Pyoderma gangrenosum $(\mathrm{PG})$ being a primary sterile neutrophilic dermatosis, the etiology is still uncertain and it rarely affects children. A high index of suspicion is essential to execute its prompt treatment. To the best of our knowledge this is the first reported case in our country

\section{KEYWORDS}

Pyoderma gangrenosum, external ear, limited setting, Tanzania

KEY CLINICAL MESSAGE 
Involvement of the external ear by PG is a rare encounter and steroids remain to be the main stay in its treatment

\section{INTRODUCTION}

Pyoderma gangrenosum $(\mathrm{PG})$ refers to a primary sterile neutrophilic dermatosis with an idiopathic etiology that rarely affects children. ${ }^{1,2}$ Involvement of the external ear is a rare encounter though its rarity does not seem to determine its inherent behavior with regard to characteristics of the ulcer and response to treatment. ${ }^{3-5}$ The diagnosis of PG remains to be clinical especially in resource-limited settings and there is no consensus to date on its gold-standard treatment. The patient in this case report was diagnosed clinically and responded well to steroids among other prescribed medications and with no relapse after six-months follow-up. A high index of suspicion is essential to execute its prompt treatment. The objective of this case report was to report an unusual case of involvement of external ear by PG and to the best of our knowledge this is the first reported case in our country.

\section{CASE REPORT}

A 3-year old male child presented to one of the remote dispensaries with a history of 6-months ulcerative lesions involving the external ear. Lesions were painful and were accompanied by a history of recurrent non-foul smelling ipsilateral otorrhoea. The kid had no history of fever or night sweats, history of trauma involving the external ear or gastrointestinal complaints.

On examination, he was malnourished and the ear had necrotic skin overlying the pinna and multiple papules with some being pus filled.

The patient was subjected to laboratory work ups; Hemoglobin was $13 \mathrm{~g} / \mathrm{dl}$, HIV negative. Histopathological analysis of the lesions and immunological work ups such as purified protein derivative, venereal disease research laboratory were not done due to remoteness of health facility. Serum albumin and globulin were not ordered because of the same reason despite the child being malnourished. Clinical diagnosis of PG of the external ear was made. (Figure 1 and 2)

The patient received; Intramuscular triamcinolone $40 \mathrm{mg}$ stat, prednisolone tablets where $10 \mathrm{mg}$ once daily for 7 days was provided and then tapered to $5 \mathrm{mg}$ once daily for 7 days, betamethasone cream which was applied topically over the ulcerated external ear, syrup ampicillin+cloxacillin 250mg administered 8-hourly for 7 days and boric acid ear drops were applied 4hourly for 7 days. The patient was followed up for 6 -months with no recurrence. (Figure 3)

\section{DISCUSSION}

Pyoderma gangrenosum being a rare disease was first elaborated in 1930 and described as a chronic destructive inflammatory, noninfectious skin disease with recurrence tendency. ${ }^{1,2} \mathrm{PG}$ lesions typically present as painful, erythematous pustules, bullae, or nodules., ${ }^{1,3-6}$ They may evolve into necrotic plaques with raised edges or into violaceous deep ulcers with undermined bluish borders, healing with atrophic or cribriform scars. Such description matches with what was seen in our case report. ${ }^{1,2,4-8}$

PG affects mainly adults aged between 20 to 50 years with female predilection. It account for only $4 \%$ of cases in children and adolescents with a higher incidence of head and perineal involvement. PG has preponderance of pustular lesions, and a frequent history of trauma. ${ }^{1-3,5,6,8-10}$ The commonest presentation of PG in children is disseminated ulcerative lesions. ${ }^{1,6,8}$

The actual pathogenesis of PG has remained to be idiopathic though it has been postulated to be a neutrophilic disorder characterized by dysregulation of the immune system, such as overexpression of growth factors and interleukins, especially interleukin (IL)-1, (IL)-6 and (IL)-8 and tumor necrosis factor (TNF)-a. ${ }^{10}$

Though the etiology has been idiopathic in majority of cases, in about fifty-percent of cases, it's associated with neoplasms and/or systemic inflammatory illnesses, such as Behcet' disease, ulcerative colitis and Crohn's disease. ${ }^{4,7}$ More recently, PG has been associated with novel drug therapies like pegfilgrastim. ${ }^{2}$ 
The diagnosis of pediatric PG is often delayed, with an average of 2 months due to its lower prevalence among children and because it can be misdiagnosed with other ulcerative diseases. ${ }^{1,2,6}$ This correlate with what was seen in this case where the diagnosis was delayed for 6-months.

Though there are no histopathological findings specific to PG, but skin biopsy should be performed to rule out other etiologies. ${ }^{1.3,4,6}$ In resource limited settings, its diagnosis remains to be clinical.

Once the diagnosis of pediatric PG is confirmed, clinicians are recommended to identify for an underlying etiology as the disease can be the initial presentation of a systemic disorder. ${ }^{1}$ This was a challenge to our case since the patient was residing at a resource-limited setting.

The head and neck region may be affected by PG, although to date only few cases of ear involvement have been reported. ${ }^{1,6,8}$ This is the first novel case reported in our country involving the external ear.

To date, there is no gold standard treatment or published algorithm for choice of therapy for pediatric PG though treatment should be tailored according to the underlying etiology. It includes systemic steroids, corticosteroid sparing agents such as dapsone and cyclosporine, and TNF-alpha inhibitors such as adalimumab and infliximab. Response to treatment is high with cure rates reaching $90 \%{ }^{6,10}$

Our patient achieved a rapid response when treated with steroids with no recurrence after 6 -months of follow up thus laying emphasis on the role of steroids in PG. Auricular cases previously reported showed good response to the classical therapeutic strategy based on the use of corticosteroids or neutrophil chemotaxis inhibitors and thus use of these drugs as first choice. No cases of auricular PG treated with anti-TNF??biologic agents have been reported from the available literatures. Thus, its value in managing refractory cases of auricular PG is unknown, as already shown in PG of other sites. ${ }^{10}$ Despite the good response to steroids manifested in auricular PG cases, yet clinicians must expect episodes of flare-up in some patients, as usually observed in most cases of typical pyoderma. No flare has been reported in this case report.

\section{CONCLUSION}

A high index of suspicion is of paramount importance towards prompt diagnosis and treatment of PG. Clinical diagnosis remains to be the main stay in resource limited settings similar to what can be depicted from this case report. Steroids have been found be the cornerstone in management of PG in our case report similar to what has been reported in other parts of the world.

\section{ACKNOWLEDGEMENT}

The parent of the child is highly acknowledged for providing a written informed consent to publish the case report.

\section{CONFLICTS OF INTEREST}

None declared

\section{AUTHOR CONTRIBUTIONS}

ZSA: Collected information for the case and drafted the initial version of the manuscript. EPT: Drafted the initial version of the manuscript. AAK: Critical feedback and editing of the manuscript. All authors read and approved the final version to be published in Clinical Case Reports

\section{ACKNOWLEDGMENTS}

\section{REFERENCES}

1. Sousa MC, Lemos ÉF, De Morais OO, Coutinho AS, Gomes CM. Pyoderma gangrenosum leading to bilateral involvement of ears. The Journal of clinical and aesthetic dermatology. 2014 Jan;7(1):41.

2. Gameiro A, Pereira N, Cardoso JC, Gonçalo M. Pyoderma gangrenosum: challenges and solutions. Clinical, cosmetic and investigational dermatology. 2015;8:285. 
3. Al-Rimawi HS, Abuekteish FM, Daoud AS, Oboosi MM. Familial pyoderma gangrenosum presenting in infancy. European journal of pediatrics. 1996 Sep 1;155(9):759-62.

4. Wcisło-Dziadecka D, Bergler-Czop B, Brzezińska-Wcisło L, Arasiewicz H. Extensive phlegmon and pyoderma gangrenosum: diagnostic difficulties. Advances in Dermatology and Allergology/Postpy Dermatologii i Alergologii. 2015 Feb;32(1):46.

5. Kędzierska K, Sindrewicz K, Smektała T, Wiśniewska M, Masiuk M, Staniszewska E, Sporniak-Tutak K, Gołembiewska E, Ciechanowski K. Wegener's granulomatosis and pyoderma gangrenosum-rare causes of facial ulcerations. Advances in Hygiene \& Experimental Medicine/Postepy Higieny i Medycyny Doswiadczalnej. 2016 Jan $1 ; 70$.

6. Paramkusam G, Meduri V, Gangeshetty N. Pyoderma gangrenosum with oral involvement-case report and review of the literature. International journal of oral science. 2010 Jun;2(2):111-6.

7. Yamamoto T. Pyoderma gangrenosum: An important dermatologic condition occasionally associated with rheumatic diseases. World Journal of Rheumatology. 2015 Jul 12;5(2):101-7.

8. Snyder RA. Pyoderma gangrenosum involving the head and neck. Archives of dermatology. 1986 Mar 1;122(3):295-302.

9. Khandpur S, Mehta S, Nagi Reddy BS. Pyoderma gangrenosum in two siblings: a familial predisposition. Pediatric dermatology. 2001 Aug;18(4):308-12.

10. Wollina U. Pyoderma gangrenosum-a review. Orphanet journal of rare diseases. 2007 Dec 1;2(1):19.

\section{LIST OF FIGURE CAPTIONS}

Figure 1: Showing necrotic external ear with some pustules before treatment initiation.

Figure 2: Showing necrotic external ear with some mutilated sites prior treatment.

Figure 3: Showing complete healing of the external ear after 6-months follow up 
Figure 1: Showing necrotic external ear with some pustules before treatment initiation.

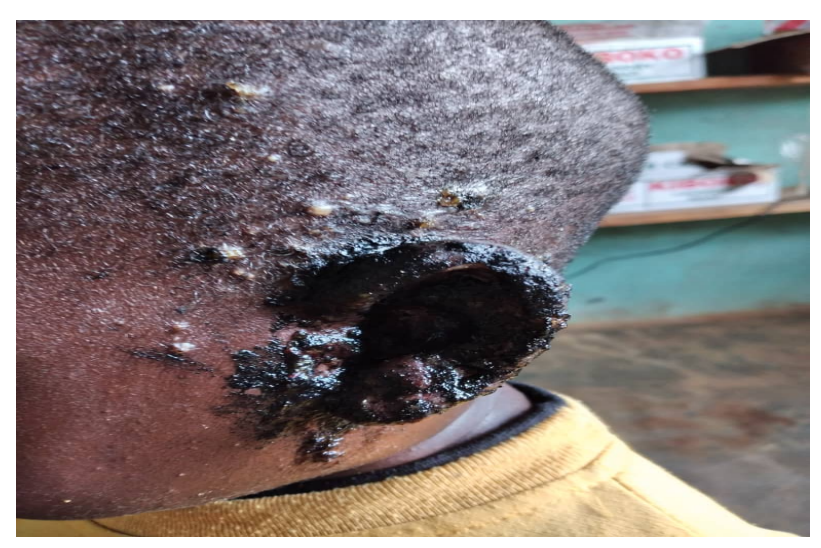


Figure 2: Showing necrotic external ear with some mutilated sites prior treatment

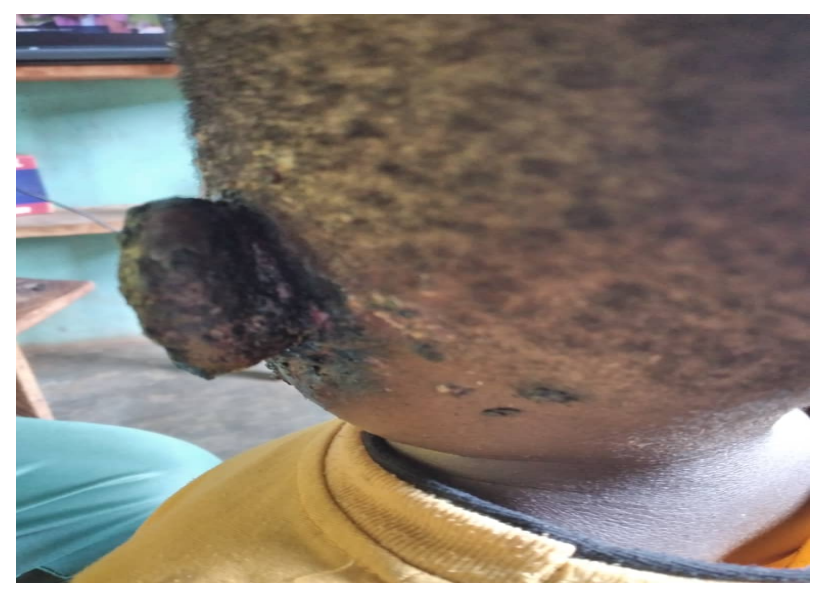


Figure 3: Showing complete healing of the external ear after 6-months follow up

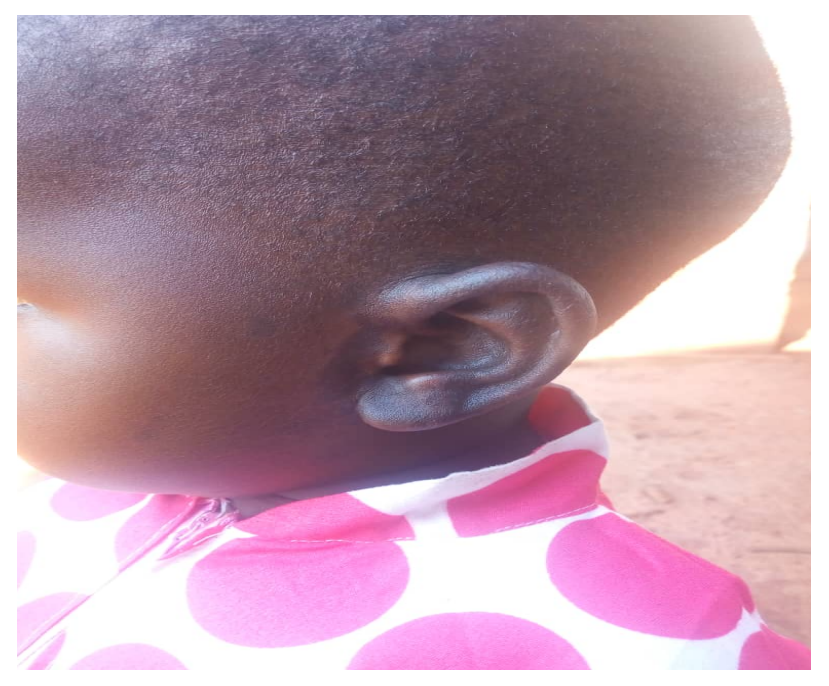

